\title{
Mortalidade materna no Estado do Rio de Janeiro em 2000 e 2011
}

\author{
Alexandre J. B. Trajano, ${ }^{1,2}$ Denise L. M. Monteiro, ${ }^{1,3^{*}}$ Luciane S. Tavares, ${ }^{4}$ Paula A. R. Alves, ${ }^{5}$ \\ Thayanna A. P. Gonçalves 5
}

\section{Resumo}

Neste artigo, apresentamos uma discussão sobre a morte materna, a partir da análise dos óbitos de gestantes e puérperas, ocorridos no Estado do Rio de Janeiro nos anos 2000 e 2011, com o objetivo de conhecer suas principais causas e analisar a relação com as variáveis sociodemográficas destas mulheres. Foram identificadas 197 mortes maternas no Estado do Rio de Janeiro no ano de 2000 e 164 em 2011. Esta cifra correspondeu à razão de mortalidade materna (RMM) de 76,0/100.000 nascidos vivos (NV) e 74,3/100.000 NV, respectivamente. O óbito materno direto apresentou redução às custas do aumento do óbito materno indireto nesse período, embora continue a ser o tipo mais frequente. A síndrome hipertensiva da gravidez persiste como a principal causa de morte materna, apesar de mostrar tendência de declínio (RMM = 20,84 em 2000 e 11,79 em 2011). A segunda causa é hemorragia obstétrica que apresentou discreto aumento no período (9,26 para 10,88/100.000 $\mathrm{NV}$ ), seguida do aborto e da infecção. Verificou-se que o risco de morte materna aumenta de forma diretamente proporcional à idade, em especial no grupo de mulheres acima de 40 anos e inversamente proporcional à escolaridade da gestante. Em relação à etnia/cor, não houve modificação entre 2000 e 2011, evidenciando que as mulheres de cor preta apresentam risco cinco vezes maior de morte no período da gravidez, parto e puerpério do que as de cor branca. Sendo a morte materna um evento-sentinela, indicativo da qualidade deficiente da assistência à saúde prestada à população, concluiu-se que o Estado do Rio de Janeiro ainda precisa aprimorar a assistência durante o ciclo grávido-puerperal, visto que a taxa de mortalidade materna apresentou redução muito discreta, sendo ainda muito elevada.

Descritores: Morte materna; Qualidade da assistência à saúde; Sistemas de informação; Mortalidade materna.

\section{Abstract \\ Maternal mortality in the state of Rio de Janeiro in 2000 and 2011}

In this article, we talked about maternal death, from the analyses of the deaths of pregnant and postpartum women, occurred in the State of Rio de Janeiro in 2000 and 2011, we mainly focused the causes and analyzed the social-economic characteristics of these women. Were identified 197 maternal deaths in Rio de Janeiro State in 2000 and 164 in 2011. This value corresponded to the maternal mortality ratio (MMR) of 76.0/100,000 live births (LB) and 74.3/100,000 live births, respectively. The directly maternal death decreased, due to an
1. Departamento de Ginecologia e Obstetrícia. Faculdade de Ciências Médicas. Universidade do Estado do Rio de Janeiro. Rio de Janeiro, RJ, Brasil.

2. Departamento de Ginecologia e Obstetrícia. Escola de Ciências de Saúde. Universidade do Grande Rio. Rio de Janeiro, RJ, Brasil.

3. Departamento de Ginecologia e Obstetrícia. Centro Universitário Serra dos Órgãos. Teresópolis. Rio de Janeiro, RJ, Brasil.

4. Secretaria Executiva. Comitê Estadual de Prevenção e Controle de Morte Materna e Perinatal do Rio de Janeiro. Departamento de Ginecologia e Obstetrícia. Fundação Técnico-Educacional Souza Marques. Rio de Janeiro, RJ, Brasil.

5. Departamento de Ginecologia e Obstetrícia. Programa de Residência Médica em Ginecologia e Obstetrícia. Universidade do Estado do Rio de Janeiro. Rio de Janeiro, RJ, Brasil.

\section{*Endereço para correspondência:}

Núcleo Perinatal, HUPE, UERJ

Endereço: Av. Prof. Manuel de Abreu, 500

Rio de Janeiro, RJ, Brasil. CEP: 20550-170

E-mail: flaviapfleming@gmail.

Revista HUPE, Rio de Janeiro, 2015;14(2):47-53

doi: 10.12957/rhupe.2015.18417

Recebido em 11/09/2014. Aprovado em 13/01/2015.

increase of indirect maternal death in this period, although it remains the most frequent type. The hypertensive disorder of pregnancy persists as the leading cause of maternal death, despite its tendency to decline ( $\mathrm{RMM}=20.84$ in 2000 and 11.79 in 2011). The second cause is bleeding which showed a slight increase in the period ( 9.26 to $10.88 / 100,000$ LB), followed by abortion and infection. It was found that the risk of maternal death increases proportionally with age, especially in the group of women over 40 years and inversely proportional to the schooling of pregnant patients. Regarding color/ethnicity, there was no change between 2000 and 2011, showing that black women have five times higher risk of death during pregnancy, delivery and postpartum period than white. As maternal death is a sentinel event, indicative of the deficient quality of health care provided to the population, it was concluded that the State of Rio de Janeiro still need to improve care during pregnancy and postpartum period, considering that maternal mortality showed very slight decrease, being still very high.

Keywords: Maternal death; Quality of health care; Information systems; Maternal mortality. 


\section{Artigo de revisão}

\section{Resumen}

\section{Mortalidad materna en el Estado de Río de Janeiro en 2000 y 2011}

En este artículo, se presenta una discusión sobre la muerte materna, a partir del análisis de los óbitos de mujeres embarazadas y puérperas, ocurridos en el estado de Río de Janeiro en 2000 y 2011, con el objetivo de conocer las principales causas y analizar la relación con las variables sociodemográficas de estas mujeres. Se identificaron 197 muertes maternas en el estado de Río de Janeiro en el año 2000 y 164 en 2011. Esta cifra corresponde a la razón de mortalidad materna (RMM) de 76,0 /100.000 nacidos vivos (NV) y 74,3 /100.000 $\mathrm{NV}$, respectivamente. El óbito materno directo disminuyó a costa del aumento del óbito materno indirecta en ese período, aunque sigue siendo el tipo más común. El síndrome hipertensivo del embarazo continua siendo la principal causa de muerte materna, a pesar de mostrar tendencia descendente

\section{Introdução}

Globalmente, estima-se que em 2011 tenham ocorrido aproximadamente 273.500 mortes maternas, ${ }^{1}$ número inferior ao computado em 2010 (287.000 casos). ${ }^{2}$ Comparando-se com o ano de 1990, quando ocorreram 409.100 mortes maternas, houve diminuição mundial de $33 \%{ }^{1}$

O declínio da mortalidade materna tem sido associado à melhoria do acesso à educação, à maior renda familiar, aos avanços da assistência obstétrica e à diminuição das taxas de gravidez, ${ }^{3}$ o que justifica a grande maioria dos casos de mortes maternas ocorrerem nos países em desenvolvimento. ${ }^{2,3}$ A África Subsaariana (56\%) e o Sul da Ásia (29\%) foram responsáveis por 85\% do total (245.000 mortes maternas em 2010). Dois países respondem por um terço das mortes maternas globais: Índia, com 19\% (56.000) e Nigéria, com 14\% (40.000). ${ }^{2}$ Em 2011, os países que apresentaram as maiores taxas de mortalidade materna foram Eritreia, Libéria, Burundi e Afeganistão, sendo as menores verificadas na Islândia e Áustria. ${ }^{1}$

A razão de mortalidade materna (RMM) mundial, em 2010, foi de 210/100.000 nascidos vivos (NV), correspondendo à quase metade da RMM de 1990 (400/100.000 NV). Mesmo assim, ainda é15 vezes maior nos países em desenvolvimento do que nos desenvolvidos. ${ }^{2,4}$ Lozano et al. (2011) relataram declínio das taxas de mortalidade materna e infantil entre 1990 e 2011, constatando que na maioria dos países (106 de 193 países) a taxa de declínio se acelerou entre 2000 e $2011 .{ }^{1}$

Apesar desta queda, as estimativas globais revelam
( $R M M=20,84$ en 2000 y 11,79 en 2011). La segunda causa es la hemorragia obstétrica que presentó un ligero aumento en el período (9,26 a 10,88 /100.000 NV), seguida por el aborto y la infección. Se verificó que el riesgo de muerte materna aumenta en proporción directa con la edad, especialmente en el grupo de mujeres mayores de 40 años e inversamente proporcional a la escolaridad de la gestante. En cuanto a la etnia/color, no hubo cambios entre 2000 y 2011, demostrando que las mujeres negras tienen cinco veces más riesgo de muerte durante el período de embarazo, parto y posparto que las mujeres blancas. Siendo la mortalidad materna un evento centinela, indicio de la mala calidad de la atención médica prestada a la población, se concluyó que el Estado de Río de Janeiro todavía tiene que mejorar la atención durante el ciclo grávido-puerperal, ya que la tasa de mortalidad materna presentó una disminución muy ligera, siendo todavía muy elevada.

Palabras clave: Muerte materna; Calidad de la atención de salud; Sistemas de información; Mortalidad materna.

que os resultados atingidos serão insuficientes para alcançar, na maioria dos países, as Metas de Desenvolvimento do Milênio de reduzir a RMM em 75\% entre 1990 e 2015. ${ }^{1,2}$

O Ministério da Saúde estima que, no Brasil, a RMM em 1990 era de 140/100.000 NV.Para alcançar esta meta de redução, nosso país precisaria atingir 35 mortes maternas/100.000 NV até o ano de 2015. Em 2000, a RMM caiu quase pela metade, alcançando 73,3/100.000 e, a partir dessa época, registra-se desaceleração do ritmo de redução da mortalidade materna no Brasil, ${ }^{5}$ chegando a 64,8/100.000 no ano de $2011,{ }^{6}$ constituindo questão de saúde pública da maior relevância e desafio constante na prática obstétrica. No Estado do Rio de Janeiro, a taxa de morte materna também apresenta tendência de estagnação, persistindo alta durante este período.

Neste trabalho, apresentamos uma discussão sobre a morte materna, a partir da análise dos óbitos de gestantes e puérperas ocorridos no Estado do Rio de Janeiro no ano de 2000 (197 casos) e 2011 (164 casos), ${ }^{6}$ visando melhor entendimento das suas principais causas e análise da relação com as variáveis sociodemográficas dessas mulheres. Desta forma, somos de opinião que o estudo da mortalidade materna é prioritário para o entendimento das condições de saúde da população e na formulação de políticas voltadas para a promoção e proteção da saúde da mulher.

\section{Definição de morte materna (óbito materno)}

A morte materna é definida como o óbito de uma 
mulher durante a gestação ou num período de 42 dias após o término da gestação, independentemente de duração ou da localização da gravidez. A causa da morte pode estar relacionada ou ser agravada pela gravidez ou por medidas referentes a ela, porém não em razão de causas acidentais ou incidentais:?

a) morte materna obstétrica - dividida em direta e indireta;

b) morte materna obstétrica direta é a que ocorre por complicações obstétricas durante a gravidez, parto ou puerpério, por intervenções, omissões, tratamento incorreto ou cadeia de eventos resultantes de quaisquer destas causas. Corresponde aos óbitos codificados na CID-10 como: O00.0 a 08.9; $\mathrm{O} 11$ a O23.9; O24.4; O26.0 a O92.7; D39.2; E23.0; F53 e M83.0;

c) morte materna obstétrica indireta é a resultante de doenças que existiam antes da gestação ou que se desenvolveram neste período, não provocadas por causas obstétricas diretas, mas agravadas pelos efeitos fisiológicos da gravidez. Corresponde aos óbitos codificados na CID-10 como: O10.0 a O10.9; O24.0 a O24.3; O24.9; O25; O98.0 a O99.8; A34; B20 a B24. Os óbitos codificados como O95 devem ser relacionados à parte, como morte obstétrica não especificada;

d) morte materna não obstétrica é a resultante de causas incidentais ou acidentais não relacionadas à gravidez e seu manejo. É também chamada de morte não relacionada. Estes óbitos não são incluídos no cálculo da razão de mortalidade materna;

e) morte materna tardia é a morte de uma mulher devido a causas obstétricas, diretas ou indiretas, que ocorre num período superior a 42 dias e inferior a um ano após o fim da gravidez (CID O96);

f) morte materna declarada - quando as informações registradas na Declaração de Óbito (DO) permitem classificar o óbito como materno;

g) morte materna não declarada - quando apenas com os dados obtidos nas investigações é que se descobre tratar-se de morte materna;

h) morte materna presumível ou mascarada - aquela cuja causa básica, relacionada ao estado gravídicopuerperal, não consta na DO por falhas no preenchimento. Ocorre quando se declara como fato ocasionador do óbito apenas a causa terminal ou a lesão que sobreveio na sucessão de eventos que culminou com a morte. Desta forma, oculta-se a causa básica e impede-se a identificação do óbito materno. ${ }^{8}$

\section{Etiologia da morte materna}

Ao longo das últimas décadas, as causas mais frequentemente relacionadas aos óbitos maternos têm sido a doença hipertensiva específica da gravidez, a hemorragia, a infecção, o parto obstruído e as complicações decorrentes do abortamento realizado em condições desfavoráveis (unsafe abortion). ${ }^{9}$ Estudos recentes mostram que não houve mudanças significativas quanto à importância destes fatores etiológicos. ${ }^{1,5,6}$

A hemorragia é a principal causa obstétrica direta de mortalidade materna na África (30,8\%) e na Ásia (33,9\%). A América Latina segue o padrão da maioria dos países de alta renda, tendo os distúrbios hipertensivos como principal causa. $3,5,10$

A identificação precisa da causa da morte materna constitui um dos aspectos mais importantes e complexos do tema. Com frequência, o óbito é resultado da interação de múltiplos fatores sociais, econômicos e adequada atenção à saúde da mulher. Em algumas situações estes óbitos são inevitáveis por condições próprias da gravidade da condição obstétrica. Considerando apenas os aspectos patológicos ou fisiopatológicos envolvidos, condições como a anemia crônica, a desnutrição, as infecções, as hemorragias e os quadros hipertensivos frequentemente agem de forma sinérgica na deterioração da homeostasia da gestante ou puérpera que termina por falecer. ${ }^{8}$

\section{A mortalidade materna no Estado do Rio de Janeiro}

Considerando todas as notificações e o resultado da busca ativa, foram identificadas 197 mortes maternas no Estado do Rio de Janeiro no ano de 2000 e 164 mortes em 2011. Esta cifra correspondeu à razão de mortalidade materna de 76,0/100.000 NV e 74,3/100.000 NV, respectivamente. A distribuição proporcional segundo o tipo de óbito materno no Estado do Rio de Janeiro nos anos de 2000 e 2011 encontra-se detalhada na tabela 1 . O óbito materno direto, em que pese ser mais frequente, teve suas taxas reduzidas. Já o indireto apresentou aumento da sua frequência neste período.

Na tabela 2, encontram-se os códigos de óbitos maternos da CID 10 que compuseram os agrupamentos de causas que foram utilizados para avaliação da razão de morte materna no Estado do Rio de Janeiro entre os anos de 2000 a 2011.

No que tange à etiologia, a síndrome hipertensiva da gravidez constitui a principal causa de morte ma- 


\section{Artigo de revisão}

terna, fato esse que vem se mantendo durante todo o período referido. A tendência ao declínio evidenciada pela $\mathrm{RMM}=$ 20,84 em 2000 e 11,79 em 2011 pode estar associada à maior divulgação dos protocolos de atendimento às gestantes portadoras de síndromes hipertensivas, o que incluiu a implantação do cartaz de Diagnóstico e Tratamento da Hipertensão Arterial no Estado do Rio de Janeiro desde 2004. A segunda causa é hemorragia obstétrica, que apresentou discreto aumen-

Tabela 1. Distribuição proporcional segundo o tipo de óbito materno no Estado do Rio de Janeiro (2000 e 2011).

\begin{tabular}{c|c|c|c|c|}
\hline $\begin{array}{c}\text { Tipo de óbito } \\
\text { materno }\end{array}$ & $\begin{array}{c}\mathbf{2 0 0 0} \\
\mathbf{n}\end{array}$ & $\mathbf{\%}$ & $\begin{array}{c}\mathbf{2 0 1 1} \\
\mathbf{n}\end{array}$ & $\mathbf{\%}$ \\
\hline Direto & 129 & 65,48 & 94 & 57,32 \\
\hline Indeterminado & 15 & 7,61 & 17 & 10,37 \\
\hline Indireto & 53 & 26,9 & 53 & 32,32 \\
\hline Total & 197 & 100 & 164 & 100 \\
\hline
\end{tabular}

Fonte: Ministério da Saúde. Secretaria de Vigilância em Saúde (SVS): Sistema de Informações sobre Mortalidade (SIM) e Sistema de Informações sobre Nascidos Vivos (Sinasc).

Tabela 2. Grupo das causas de morte materna, segundo a CID-MM.

\begin{tabular}{|c|c|}
\hline Grupos de causas & CID-10 \\
\hline Gravidez ectópica & $000-000.9$ \\
\hline $\begin{array}{l}\text { Gravidez que termina em } \\
\text { aborto }\end{array}$ & 002-007.9 \\
\hline $\begin{array}{l}\text { Transtornos hipertensivos da } \\
\text { gravidez }\end{array}$ & 011-016 \\
\hline Hemorragia obstétrica & $\begin{array}{l}\text { O20-O20.9; O43-043.2; } \\
\text { O44-O44.1; O45-045.9; } \\
\text { O46-O46.9; O62.1-O62.2; } \\
\text { O67-067.9; O70.0-070.9; } \\
\text { O71.0-071.4; O71.7; } \\
\text { O72-072.3 }\end{array}$ \\
\hline $\begin{array}{l}\text { Infecção relacionada à } \\
\text { gravidez }\end{array}$ & $\begin{array}{l}\text { O23-023.9; O41.1; 075.3; } \\
\text { O85; 086-086.8; 091-091.2 }\end{array}$ \\
\hline Complicações de manejo & $\begin{array}{l}\text { O29-O29.3; O29.5-O29.9; } \\
\text { O74-O74.4; O74.6-074.9; } \\
\text { O75.4; O89-089.3; } \\
\text { O89.5-089.9; 090.0-090.2 }\end{array}$ \\
\hline Tétano na gravidez & A34 \\
\hline Óbito materno indireto & $\begin{array}{l}\text { O10-010.9; } \\
\text { O24-024.3; 024.9; } \\
\text { 098-098.9; 099-099.8 }\end{array}$ \\
\hline Causas indeterminadas & 095 \\
\hline
\end{tabular}

Fonte: Tavares LS, 2013. ${ }^{11}$ to no período $(9,26$ para $10,88 / 100.000 \mathrm{NV})$, seguida do aborto e da infecção. Em 2009, houve incremento das causas indiretas em grande parte devido à epidemia de H1N1. Mesmo desconsiderando-se este ano atípico, observa-se neste período um aumento das mortes maternas devido a causas indiretas. Na tabela 3, as causas encontram-se referidas como razão de mortalidade materna (RMM/100.000 NV), e a representação gráfica desta tabela, que mostra os quatro principais grupos de óbito materno, encontra-se na figura 1.

Ao analisarmos as razões de mortalidade materna, verificamos que sua relação com a idade é diretamente proporcional, em especial no grupo de mulheres acima de 40 anos (480/100.000). Neste grupo, a taxa de mortalidade materna foi 11 vezes superior à do grupo mais jovem, de dez a 19 anos (42/100.000). Em 2011, houve decréscimo da RMM entre as mulheres com mais de 40 anos, contudo ainda permanece a faixa etária de maior risco de morte materna (Tabela 4).

Quanto à escolaridade, observamos em 2000, maior número de casos na faixa de quatro a sete anos de estudo, o que corresponde ao nível fundamental incompleto. Quando consideramos a razão de mortalidade, verificamos que existe clara relação, inversamente proporcional ao tempo de estudo, variando de 193,5/100.000 entre as que não estudaram a 26,5/100.000 entre as que tinham mais de 11 anos de escolaridade. Esta significativa diferença de cinco vezes reflete a importância da escolaridade como indicador das condições de vida da mulher. No ano de 2011, esta diferença foi 11 vezes maior (Tabela 5 ).

Em relação à etnia/cor, não houve modificação do risco de morte materna entre 2000 e 2011, evidenciando que o risco da mulher da cor preta se manteve cinco vezes maior que a de cor branca (Tabelas 6 e 7).

\section{Conclusões}

As síndromes hipertensivas, as hemorragias maternas, assim como a infecção puerperal e o abortamento são as complicações que, com mais frequência, estão associadas à morte materna. O risco de morte materna aumenta de forma diretamente proporcional à idade e inversamente proporcional à escolaridade. As mulheres de cor preta apresentam risco cinco vezes maior de morte no período da gravidez, parto e puerpério. A taxa de mortalidade materna (TMM) verificada no Estado do Rio de Janeiro ao longo do período apresentou redução muito discreta, é ainda muito elevada e indica o comprometimento da qualidade da assistência obstétrica 
Tabela 3. Distribuição das principais causas de morte materna entre 2000 e 2011 no Estado do Rio de Janeiro (RMM/100.000 NV).

\begin{tabular}{|c|c|c|c|c|c|c|c|c|c|c|c|c|}
\hline Causas (RMM) & 2000 & 2001 & 2002 & 2003 & 2004 & 2005 & 2006 & 2007 & 2008 & 2009 & 2010 & 2011 \\
\hline $\begin{array}{l}\text { Sínd. hipertensiva } \\
\text { da gravidez }\end{array}$ & 20,84 & 16,50 & 22,39 & 17,22 & 14,35 & 16,58 & 18,77 & 15,68 & 13,44 & 11,08 & 14,87 & 11,79 \\
\hline $\begin{array}{l}\text { Hemorragia } \\
\text { obstétrica }\end{array}$ & 9,26 & 13,20 & 12,49 & 7,75 & 10,00 & 11,21 & 8,70 & 15,22 & 8,34 & 7,85 & 10,22 & 10,88 \\
\hline Aborto & 6,56 & 6,60 & 6,46 & 9,04 & 6,09 & 4,48 & 5,49 & 4,61 & 8,34 & 6,46 & 8,36 & 5,89 \\
\hline Gravidez ectópica & 1,54 & 1,65 & & 3,01 & 0,87 & 3,14 & 1,37 & 4,61 & 0,46 & 2,77 & 2,32 & 2,27 \\
\hline $\begin{array}{l}\text { Infecção relacionada } \\
\text { à gravidez }\end{array}$ & 4,63 & 5,78 & 6,89 & 6,03 & 3,91 & 7,17 & 7,78 & 8,30 & 6,95 & 9,69 & 5,11 & 2,27 \\
\hline $\begin{array}{l}\text { Complicações de } \\
\text { manejo }\end{array}$ & 0,77 & 1,65 & 0,43 & 0,43 & 0,87 & 0,45 & 0,92 & 2,31 & 0,93 & 1,38 & 0,46 & 0,45 \\
\hline $\begin{array}{l}\text { Outras complicações } \\
\text { obstétricas }\end{array}$ & 6,17 & 8,66 & 7,75 & 9,04 & 12,61 & 8,96 & 10,99 & 7,38 & 11,58 & 11,08 & 12,54 & 9,07 \\
\hline Indeterminado & 5,79 & 3,30 & 2,15 & 1,72 & 1,30 & 1,34 & 1,83 & 1,38 & 2,78 & 2,31 & 4,65 & 7,71 \\
\hline Indireto & 20,45 & 14,03 & 15,50 & 13,78 & 19,14 & 9,86 & 19,23 & 19,83 & 17,14 & 41,08 & 25,09 & 24,03 \\
\hline
\end{tabular}

Notas: MS/SVS/DASIS - Sistema de Informações sobre Mortalidade - SIM.

MS/SVS/DASIS - Sistema de Informações sobre Nascidos Vivos - SINASC.

Figura 1. Representação gráfica da tabela 3.

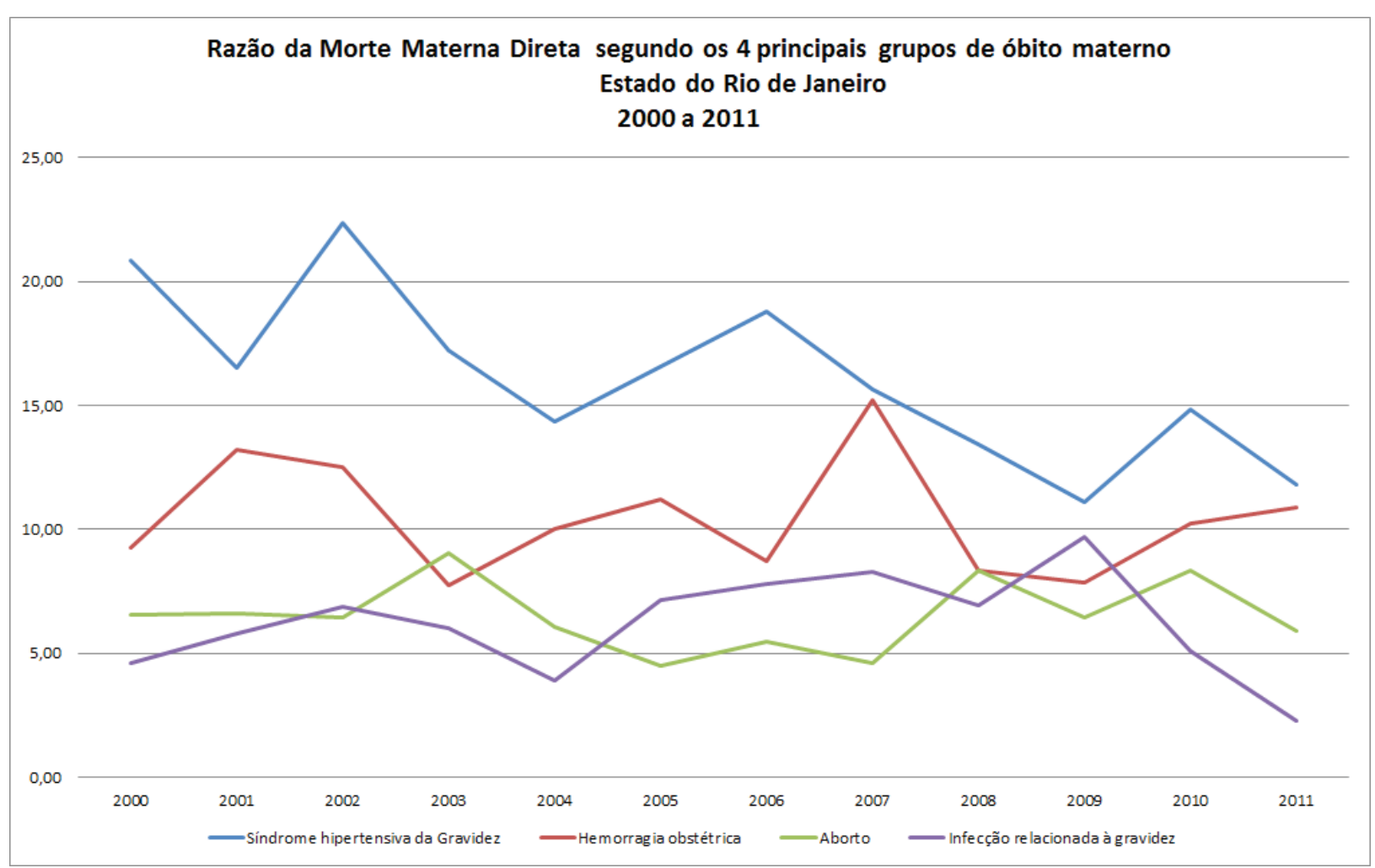

Notas: MS/SVS/DASIS - Sistema de Informações sobre Mortalidade - SIM.

MS/SVS/DASIS - Sistema de Informações sobre Nascidos Vivos - SINASC. 


\section{Artigo de revisão}

Tabela 4. Distribuição da idade dos óbitos maternos em 2000 e 2011 no Estado do Rio de Janeiro (RMM/100.000 NV).

\begin{tabular}{|c|c|c|c|c|c|c|}
\hline \multirow{2}{*}{ Idade } & \multicolumn{3}{|c|}{2000} & \multicolumn{3}{|c|}{2011} \\
\hline & $\mathbf{N}$ & $\%$ & RMN & $\mathbf{N}$ & $\%$ & RMN \\
\hline $10-14$ & 0 & 0 & 0 & 3 & 1,83 & 171,33 \\
\hline $15-19$ & 22 & 11,17 & 41,84 & 22 & 13,41 & 58,01 \\
\hline $20-24$ & 48 & 24,37 & 49,89 & 38 & 23,17 & 68,55 \\
\hline $25-29$ & 40 & 20,3 & 52,51 & 35 & 21,34 & 64,74 \\
\hline $30-34$ & 26 & 13,2 & 64,18 & 32 & 19,51 & 72,49 \\
\hline $35-39$ & 36 & 18,27 & 174,11 & 22 & 13,42 & 101,88 \\
\hline$>40$ & 25 & 12,69 & 479,94 & 12 & 7,32 & 210,97 \\
\hline Total & 197 & 100 & & 164 & 100 & \\
\hline
\end{tabular}

Fonte: MS/SVS/DASIS - Sistema de Informações sobre Mortalidade - SIM

MS/SVS/DASIS - Sistema de Informações sobre Nascidos Vivos - SINASC.

Tabela 5. Distribuição da escolaridade dos óbitos maternos em 2000 e 2011 no Estado do Rio de Janeiro (RMM/100.000 NV).

\begin{tabular}{c|c|c}
\cline { 2 - 3 } & \multicolumn{2}{c}{$\mathrm{RMN}$} \\
\hline Escolaridade & $\mathbf{2 0 0 0}$ & $\mathbf{2 0 1 1}$ \\
\hline nenhuma & 193,5 & 285,3 \\
\hline 1 a 3 anos & 123,0 & 341,9 \\
\hline 4 a 7 anos & 63,7 & 107,5 \\
\hline 8 a 11 anos & 30,4 & 47,5 \\
\hline 12 anos e mais & 26,5 & 24,5 \\
\hline
\end{tabular}

Fonte: Ministério da Saúde. SVS: SIM e Sinasc.

e das condições de saúde da população, impondo a adoção de ações conjuntas na esfera acadêmica, pela formação e capacitação profissional, e no âmbito governamental, pelo estabelecimento de políticas e provimento dos recursos necessários para a reversão desta dramática realidade. ${ }^{12}$

\section{Referências}

1. Lozano R, Wang $\mathrm{H}$, Foreman $\mathrm{KJ}$, et al. Progress towards Millennium Development Goals 4 and 5 on maternal and child mortality: an updated systematic analysis. Lancet 2011;378(9797):1139-65. doi: 10.1016/S0140-6736(11)613378. Epub 2011 Sep 19.

2. WHO, UNICEF, UNFPA, the World Bank. Trends in maternal mortality: 1990 to 2010. WHO, UNICEF, UNFPA and the World Bank estimates. Geneva: World Health Organization, 2012. Disponível em: http://www.unfpa.org/public/home/publications/ pid/10728. Acesso em 10Mar2014. [não foi possível localizar
Tabela 6. Distribuição da etnia dos óbitos maternos em 2000 e 2011 no Estado do Rio de Janeiro (RMM/100.000 NV).

\begin{tabular}{c|c|c}
\cline { 2 - 3 } & \multicolumn{2}{c}{$\mathrm{RMN}$} \\
\hline Etnia & $\mathbf{2 0 0 0}$ & $\mathbf{2 0 1 1}$ \\
Branca & 50,09909 & 45,84671 \\
\hline Preta & 254,2056 & 238,594 \\
Parda & 74,11848 & 71,67626 \\
\hline Indígena & 0 & 884,9558 \\
\hline
\end{tabular}

Fonte: Ministério da Saúde. SVS: SIM e Sinasc.

Tabela 7. Distribuição da sobremortalidade em 2000 e 2011 no Estado do Rio de Janeiro (RMM/100.000 NV).

\begin{tabular}{c|c|c}
\hline Sobremortalidade & $\mathbf{2 0 0 0}$ & $\mathbf{2 0 1 1}$ \\
\hline Preta/Branca & 5,074056 & 5,20417 \\
\hline Parda/Branca & 1,479438 & 1,563389 \\
\hline
\end{tabular}

Fonte: Ministério da Saúde. SVS: SIM e Sinasc.

a página citada nem a referência. Talvez o link já tenha sido expirado.]

3. Hogan MC, Foreman KJ, Naghavi M, Ahn SY, Wang M, et al. Maternal mortality for 181 countries, 1980-2008: a systematic analysis of progress towards Millennium Development Goal 5. Lancet 2010;375(9726):1609-23. doi: 10.1016/S01406736(10)60518-1. Epub 2010 Apr 9

4. Hill K, El Arifeen S, Koenig M, et al. How should we measure maternal mortality in the developing world? A comparison of household deaths and sibling history approaches. Bulletin of 
Alexandre J. B. Trajano e cols. • Mortalidade materna no Estado do Rio de Janeiro em 2000 e 2011

the World Health Organization, 2006,84:173-180.

5. Souza JP. Mortalidade materna no Brasil: a necessidade de fortalecer os sistemas de saúde. Rev. Bras. Ginecol. Obstet. [serial on the Internet]. 2011;33(10):273-279. http://dx.doi. org/10.1590/S0100-72032011001000001.

6. Ministério da Saúde. Secretaria de Vigilância em Saúde (SVS): Sistema de Informações sobre Mortalidade (SIM) e Sistema de Informações sobre Nascidos Vivos (Sinasc). Disponível em tabnet.datasus.gov.br/cgi/idb2012.C03b.htm. Acesso em 10Mar2014. [não foi possível localizar a página citada nem a referência. Talvez o link já tenha sido expirado.]

7. WHO. World Health Organization. Classificação Estatística Internacional de Doenças e Problemas Relacionados à Saúde. 10. rev. São Paulo: CBCD, 1995.

8. Brasil. Ministério da Saúde. Secretaria de Vigilância em Saúde. Departamento de Análise de Situação em Saúde. Guia de vigilância epidemiológica do óbito materno / Ministério da Saúde,
Secretaria de Vigilância em Saúde, Departamento de Análise de Situação em Saúde. - Brasília : Ministério da Saúde, 2009. 84 p. : il. - (Série A. Normas e Manuais Técnicos).

9. Abouzahr C, Royston E. Maternal mortality: a global factbook. Geneva, World Health Organization, 1991. 606 p.

10. U.S. Department of Health and Human Services, Health Resources and Services Administration, Maternal and Child Health Bureau. Child Health USA 2008-2009, Rockville, Maryland 2005. [referência não encontrada]

11. Tavares LS. Condições maternas: uma abordagem holística. / Luciane Santiago Tavares. -- 2013.105f. Tese (Doutorado) - Escola Nacional de Saúde Pública Sergio Arouca, Rio de Janeiro, 2013. [referência não encontrada]

12. Trajano AJB. Morte materna. /Alexandre José Baptista Trajano. 2002. 156 p. Tese (Concurso de Professor Titular) - Faculdade de Ciências Médicas da Universidade do Estado do Rio de Janeiro. Rio de Janeiro, RJ, Brasil. [referência não encontrada] 